\title{
Comunicación
}

\section{Descripción imagenológica de efusión articular en una yegua criolla colombiana}

\author{
Imaging description of joint effusion in a Colombian creole mare
}

\author{
Renso Sneider Gallego Rodriguez ${ }^{1,2}$, Diana Patricia Gómez Idarraga ${ }^{1}$
}

\section{Resumen}

\begin{abstract}
Se reporta el caso de un equino hembra, de raza criollo colombiano de 15 años, el cual se presenta con motivo de consulta por traumatismo en el aspecto medial del miembro posterior izquierdo. Se evidencia efusión sinovial severa con aumento de temperatura local y dolor al tacto. Se realiza una artrocentesis obteniendo líquido articular hemorrágico. En la evaluación radiológica se observa aumento del volumen y radiopacidad del líquido sinovial y al diagnóstico ecográfico se encuentra incremento en el grosor de la cápsula articular, con abundante efusión articular, con líquido de aspecto heterogéneo y presencia de bandas ecogénicas compatibles con fibrina. El abordaje terapéutico se realizó mediante lavado articular e infiltración con gentamicina, además del manejo del dolor y antinflamatorios por vía sistémica.
\end{abstract}

Palabras clave: efusión; equino; diagnóstico imagenológico; hemartrosis

\section{Abstract}

The case of a 15-year-old Colombian equine female, which is presented due to trauma consultation in the medial aspect of the left hind limb is reported. Severe synovial effusion is evidenced with increase in temperature and pain to the touch. An arthrocentesis is performed obtaining hemorrhagic articular fluid. In the radiological evaluation, an increase in the volume and radiopacity of the synovial fluid was observed, and the

\footnotetext{
${ }^{1}$ Grupo de Investigación en Veterinaria (GINVER), Facultad de Medicina Veterinaria, Corporación Universitaria Remington, Medellín, Colombia

${ }^{2}$ E-mail: renso.gallego@uniremington.edu.co
} 
ultrasound diagnosis revealed an increase in the thickness of the articular capsule, with abundant joint effusion, a heterogeneous-looking fluid and the presence of echogenic bands compatible with fibrin. The therapy consisted in the joint lavage and infiltration with gentamicin, whereas the pain and oedema were treated via systemic.

Key words: effusion; equine; diagnostic imaging; hemarthrosis

\section{INTRODUCCIÓN}

La artritis séptica en el equino puede llegar a afectar de forma irreversible una articulación. La causa más común de presentación en animales jóvenes es por vía hemática como consecuencia de septicemia, y en animales adultos mediante la penetración traumática en la articulación o por vía iatrogénica (Adams et al., 2010). Los signos clínicos son usualmente aumento de temperatura en la articulación afectada e inflamación, efusión y claudicación del miembro afectado (Haerdi-Landerer et al., 2010).

El diagnóstico de esta patología se lleva a cabo mediante signos clínicos, análisis radiográfico de la articulación afectada, ultrasonografía y artrocentesis con análisis del líquido sinovial (Whitcomb, 2006). La evaluación ecográfica del tarso se realiza mediante una sonda lineal de alta frecuencia (10 MHz), abordándose estructuras tales como los ligamentos colaterales, la cápsula articular, la membrana sinovial y se determina la ecogenicidad del líquido articular (Smith M y Smith R, 2008).

Los protocolos terapéuticos para equinos adultos con artritis séptica incluyen el uso de antibióticos parenterales y lavados por artrocentesis con perfusión articular, artrotomía, artroscopía y abordajes quirúrgicos (Rinnovati et al., 2018). La terapia antibiótica se puede realizar de forma sistémica utilizando fármacos como la penicilina $\mathrm{G}(20000 \mathrm{UI} / \mathrm{kg})$, gentamicina $(3 \mathrm{mg} / \mathrm{kg}$ ) y enrofloxacina $(5 \mathrm{mg} / \mathrm{kg})$, mientras que cuando se realiza el lavado articular se utilizan la gentamicina, amikacina y ceftiofur, los cuales son menos irritantes para el tejido articular (Castillo y Oliver, 2009).

\section{Descripción del Caso}

Equino hembra, criollo colombiano, de 15 años y $300 \mathrm{~kg}$ de peso vivo se presenta a consulta a la Clínica Veterinaria de la Corporación Universitaria Remington con historia reciente de traumatismo en el aspecto medial del miembro posterior izquierdo. Presenta tumoración y efusión articular, claudicación unilateral $3 / 5$.

En la evaluación clínica se encuentran dos heridas incisas de aspecto alargado longitudinal en la cara medial del metatarso y otra en la cara medial de la tibia; asimismo, aumento de tamaño en la articulación, aumento de temperatura local a la palpación y aumento del líquido sinovial con dolor focalizado a la palpación. Al desplazamiento se observan movimientos compensatorios en el tren posterior, con dificultad en el movimiento del miembro posterior izquierdo y aumento de tamaño del miembro. Al examen físico se evidencia aumento en la frecuencia respiratoria, nódulos linfáticos submandibulares reactivos, signos de dolor en el miembro e incomodidad para apoyarlo.

En la evaluación diagnóstica de la articulación se realizaron bloqueos perineurales (tibial y peroneo), siendo positivo al bloqueo peroneo disminuyendo la claudicación a un grado imperceptible. Luego se realizó la desinfección de la superficie de la articulación y 


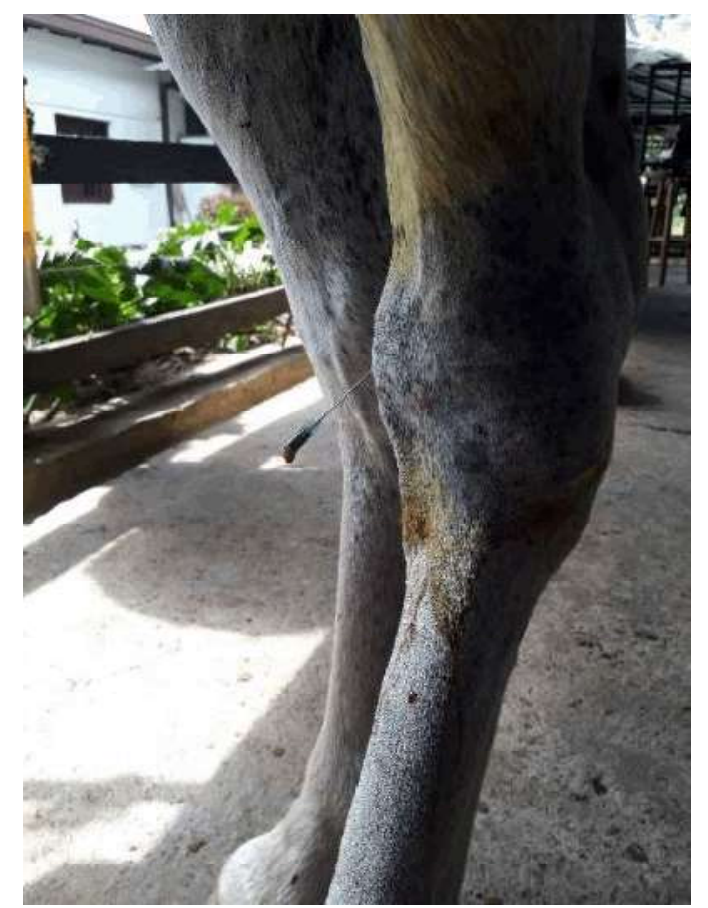

Figura 1. Articulación tarso crural con aumento de tamaño y efusión articular. La artrocentesis evidenció líquido sinovial de aspecto hemorrágico

se realizó una artrocentesis obteniéndose un líquido de color rojizo (Figura 1) y de viscosidad disminuida, lo cual puede ser compatible con la inflamación en la articulación (Lamatta, 2008). El diagnóstico presuntivo, con base al examen clínico y al examen macroscópico del líquido sinovial, fue de artritis séptica.

Ante los hallazgos obtenidos en la exploración clínica se hizo un estudio radiológico con vistas latero medial y anteroposterior del tarso izquierdo, encontrando incremento del volumen articular, aumento en el tamaño de la articulación del tarso e incremento en la densidad de los tejidos blandos periarticulares (Figura 2).

Se realizó una exploración articular mediante ecografía en la cual se evaluó la integridad articular y el líquido sinovial. Se observó incremento del líquido sinovial con cambios ecogénicos al haber estructuras de aspecto filamentoso, lo que puede ser compatible con presencia de fibrina intraarticular (Figura 3a). Asimismo, incremento del espesor de la membrana articular $(0.42 \mathrm{~mm})$ con aspecto hiperecogénico (Figura $3 \mathrm{~b}$ ), signo ecográfico compatible con sinovitis. Con base a la correlación clínica y a los hallazgos obtenidos a nivel diagnóstico, se optó como diagnóstico definitivo una artritis séptica con hemartrosis.

Se hizo una infiltración ecoguiada de la articulación con una aguja $21 \mathrm{G}$ y gentamicina en dosis de $200 \mathrm{mg}$ diluido en $5 \mathrm{ml}$ de cloruro de sodio $0.9 \%$. El tratamiento se complementó con manejo del dolor utilizando ketoprofeno a una dosis de $2.2 \mathrm{mg} / \mathrm{kg}$ vía i.m. cada $8 \mathrm{~h}$ durante dos días, y terapia antinflamatoria con dexametasona $1 \mathrm{mg} / \mathrm{kg}$ vía i.m. cada $24 \mathrm{~h}$

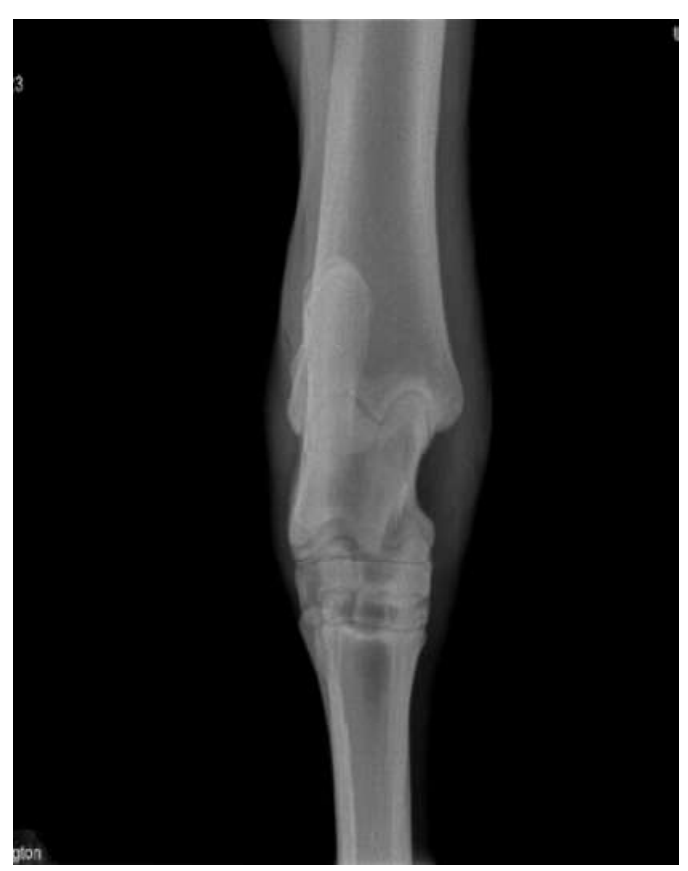

Figura 2. Estudio radiológico anteroposterior del tarso. Se observa disminución de la opacidad sobre el hueso tarso central y el tercer hueso tarsiano. Se evidencia leve aumento del tamaño y de la densidad de los tejidos blandos en la articulación hacia la región medial 

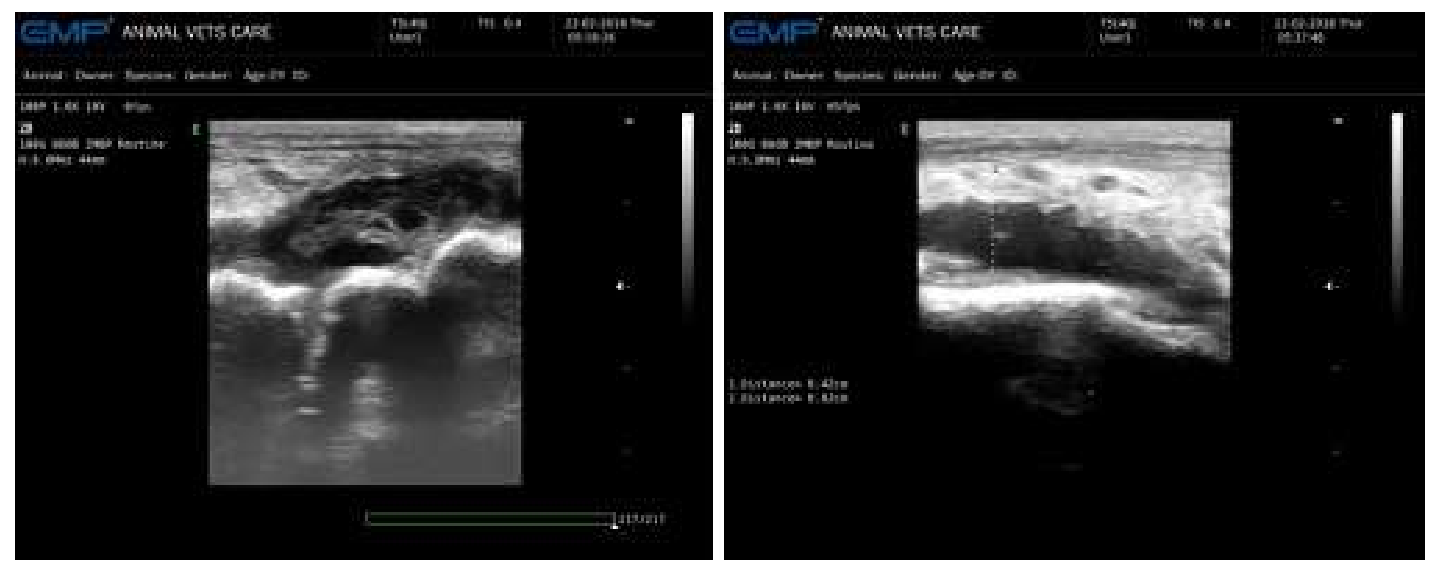

Figura 3. Estudio ecográfico. (a) Articulación tarso crural: aumento del líquido sinovial con bandas ecogénicas compatibles con fibrina. (b) Articulación intertarsiana: incremento del tamaño de la membrana sinovial $(0.42 \mathrm{~mm})$ con aumento de ecogenicidad y bordes irregulares. Incremento del líquido sinovial

por tres días. Luego de 1.5-2 días de iniciada la terapia, el paciente presentó disminución de la claudicación y la efusión era casi imperceptible. Se consideró la eficacia clínica del tratamiento a los ocho días de instaurado el tratamiento.

\section{Discusión}

La efusión articular es un hallazgo común en articulaciones diartrodiales, las cuales se relacionan con procesos inflamatorios de origen agudo, generalmente asociadas a un evento séptico. La interpretación en el diagnóstico imagenológico permite determinar algunos cambios en el tejido. En el equino, a nivel de miembros posteriores, las articulaciones del tarso son las más afectadas, y a su vez, las que más se relacionan con claudicaciones (Vanderperren et al., 2009). Las estructuras sinoviales en el equino que pueden verse afectadas por sepsis son las bursas, las vainas tendinosas y las articulaciones sinoviales, siendo la tarsocrural la articulación más afectada (Taylor, 2010; Rinnovati, 2018). Así mismo, los hallazgos más relevantes en la evaluación radiográfica de un equi- no con artritis séptica tarsiana son la osteolisis de la porción distal de la tibia a nivel fisiario y epifisiario, acompañado del aumento de la densidad del líquido articular y de los tejidos blandos (Glass y Watts, 2017), lesiones que coinciden con el presente caso.

Los signos clínicos pueden estar relacionados con aumento del tamaño de la articulación, efusión sinovial, aumento de temperatura local, dolor a la palpación y claudicación evidente (Glass y Watts, 2017). Streptococcus equi subesp. equi y Streptococcus equi subesp. zooepidemicus fueron las bacterias aisladas con mayor frecuencia en potros con artritis séptica, según Motta et al. (2017).

El diagnóstico imagenológico a nivel articular en equinos reviste gran importancia en la determinación de los posibles hallazgos. Hardy (2006) menciona que el estudio radiográfico en pacientes con artritis séptica permite correlacionar con los hallazgos clínicos presentes en el examen físico del paciente, a la vez que describe lesiones como disminución de la opacidad a nivel del hueso cortical, reacción perióstica exacerbada, aumento del 
tamaño y de la densidad de la cápsula articular, y aumento del líquido sinovial. Morton (2005) menciona, asimismo, que en pacientes adultos con historial de artritis séptica pueden encontrarse cambios articulares degenerativos debido a la erosión y posterior mineralización del cartílago articular, produciendo artrófitos o enteseofitos. En este caso en particular no se encontraron cambios degenerativos, ya que las lesiones presentes estaban más relacionadas con un estado agudo inflamatorio.

La ultrasonografía permitió determinar el aumento del tamaño de la cápsula articular $(0.42 \mathrm{~mm})$ y su aspecto hiperecogénico. Esta técnica es sensible para pacientes con distención de la cápsula articular al realizar una evaluación directa del tamaño capsular en el aspecto dorsomedial de la articulación entre el tendón del tercer peroneo y el ligamento colateral medial (Whitcomb, 2006; Sawyer et al., 2014).

\section{Literatura Citada}

1. Adams MK, Hendrickson DA, Rao S, Olea F, Bolte D. 2010. The bacteria isolated from the skin of 20 horses at a veterinary teaching hospital. J Equine Vet Sci 30: 687-695. doi: 10.1016/ j.jevs.2010.11.002

2. Castillo I, Oliver O. 2009. Artritis séptica en equinos. Reporte de 11 casos (20002004). Rev Med Vet Zoot 56: 41-52.

3. Glass K, Watts AE. 2017. Septic arthritis, physitis, and osteomyelitis in foals. Vet Clin N Am-Equine 33: 299314. doi: 10.1016/j.cveq.2017.03.002

4. Haerdi-Landerer MC, Habermacher $J$, Wenger B, Suter MM, Steiner A. 2010. Slow release antibiotics for treatment of septic arthritis in large animals. Vet J 184: 14-20. doi: 10.1016/ j.tvj1.2009.02.013
5. Hardy J. 2006. Etiology, diagnosis, and treatment of septic arthritis, osteitis, and osteomyelitis in foals. Clin Tech Equine Pract 5: 309-317. doi: 10.1053/ j.ctep.2006.09.005

6. Morton AJ. 2005. Diagnosis and treatment of septic arthritis. Vet Clin N Am-Equine 21: 627-649. doi: 10.1016/ j.cveq.2005.08.001

7. Motta R, Martins LS, Motta I, Guerra $S$, de Paula C. Bolanos CA, da Silva $R$, et al. 2017. Multidrug resistant bacteria isolated from septic arthritis in horses. Pesqui Vet Brasil 37: 325-330. doi: 10.1590/s0100-736x2017000400005

8. Rinnovati R, Bianchin B, Lanci A, Mariella J. 2018. Diagnosis, treatment, surgical, management and outcome of septic arthritis of tarsocrural joint in 16 foals. J Equine Vet Sci 9: 156-164. doi: 10.1016/j.jevs.2018.04.003

9. Sawyer CA, Talbot A, Denk D, Singer ER. 2014. Severe intermittent hind limb lameness caused by a synovial swelling on the dorsolateral aspect of the tarsus in a Dutch warmblood mare. J Equine Vet Sci 34: 436-441. doi: 10.1016/ j.jevs.2013.07.017

10. Smith M, Smith R. 2008. Diagnostic ultrasound of the limb joints, muscle and bone in horses. Clin Prac 30: 152-159. doi: 10.1136/inpract.30.3.152

11. Taylor AH, Mair TS, Smith LJ, Perkins JD. 2010. Bacterial culture of septic synovial structures of horses: does a positive bacterial culture influence prognosis? Equine Vet J 42: 213-218. doi: 10.2746/042516409X480403

12. Vanderperren $K$, Raes E, Hoegaerts M, Saunders JH. 2009. Diagnostic imaging of the equine tarsal region using radiography and ultrasonography. Vet $\mathbf{J}$ 2: 179-187. doi: 10.1016/j.tvj1.2007.08.030

13. Whitcomb M. 2006. Ultrasonography of the equine tarsus. AAEP Proc 52: 13-30. 